Iranian Journal of Mathematical Sciences and Informatics

Vol. 15, No. 1 (2020), pp 1-13

DOI: 10.21859/IJMSI.15.1.1

\title{
On Total Edge Irregularity Strength of Staircase Graphs and Related Graphs
}

\author{
Yeni Susanti $^{a, *}$, Yulia Indah Puspitasari ${ }^{b}$, Husnul Khotimah $^{c} \rrbracket$ \\ ${ }^{a}$ Department of Mathematics Universitas Gadjah Mada Indonesia \\ ${ }^{b}$ Surakarta Indonesia \\ ${ }^{c}$ Department of Mathematics Universitas Muhammadiyah Pringsewu \\ Lampung Indonesia \\ E-mail: yenimath@ugm.ac.id \\ E-mail: yuliaindahp.mail@gmail.com \\ E-mail: husnul_elsa2@yahoo.com
}

\begin{abstract}
Let $G=(V(G), E(G))$ be a connected simple undirected graph with non empty vertex set $V(G)$ and edge set $E(G)$. For a positive integer $k$, by an edge irregular total $k$-labeling we mean a function $f$ : $V(G) \cup E(G) \rightarrow\{1,2, \ldots, k\}$ such that for each two edges $a b$ and $c d$, it follows that $f(a)+f(a b)+f(b) \neq f(c)+f(c d)+f(d)$, i.e. every two edges have distinct weights. The minimum $k$ for which $G$ has an edge irregular total $k$-labeling is called the total edge irregularity strength of graph $G$ and denoted by $\operatorname{tes}(G)$. In this paper, we determine the exact value of total edge irregularity strength for staircase graphs, double staircase graphs and mirror-staircase graphs.
\end{abstract}

Keywords: Total edge irregularity strength, Staircase graphs, Double staircase graphs, Mirror-staircase graphs.

2010 Mathematics Subject Classification: 05C78, $05 \mathrm{C} 85$.

* Corresponding Author

Received 26 July 2017; Accepted 27 March 2019

(C)2020 Academic Center for Education, Culture and Research TMU 


\section{Introduction}

By Wallis [12, a labeling (or valuation) of a graph is defined as a map that relates the graph element to some numbers (usually to the positive or non negative integers). As the graph element, one can take edge set, vertex set or union of vertex set and edge set. In the case that the graph element is the union of vertex set and edge set, then the labeling is called total labeling. From Gallian [4] we observe that there are various kinds of labeling on graphs.

One sort of a graph labeling is proposed by Chartrand, et al. in [3] as the following. Assign positive integer labels to the edges of a simple connected graph of order at least 3 in such a way that the graph becomes irregular, i.e., the weights (label sums) at each vertex are distinct. What is the minimum value of the largest label over all such irregular assignments? The value is then well known as the irregularity strength of the graph. In the case that the labeling is total, and the weighted components of the graph are its edges, then the value is called as the total edge irregularity strength of the graph.

Now, let $G=(V(G), E(G))$ be a connected simple undirected graph with non empty vertex set $V(G)$ and edge set $E(G)$. Let $|E(G)|$ and $|V(G)|$ be the number of the edges and the vertices, respectively. Bača et al. 2] studied the weight $w t_{f}(x y)$ of an edge $x y$ under a total labeling $f: V(G) \cup E(G) \mapsto$ $\{1,2, \ldots, k\}$ of elements of a graph $G$ which is defined by

$$
w t_{f}(x y)=f(x)+f(x y)+f(y) .
$$

The total labeling $f$ is called an edge irregular total $k$-labeling whenever each two edges $a b$ and $c d$ have distinct weights, that are $f(a)+f(a b)+f(b) \neq$ $f(c)+f(c d)+f(d)$. The minimum $k$ for which $G$ has an edge irregular total $k$-labeling is denoted by $\operatorname{tes}(G)$ and is called the total edge irregularity strength of graph $G$.

The lower bound of the total edge irregularity strength of any graph is given by Bača et al. 22 as follows.

Theorem 1.1. 2] For any graph $G$ which maximum vertex degree is $\Delta(G)$, it follows that tes $(G) \geq \max \left\{\left\lceil\frac{|E(G)|+2}{3}\right\rceil,\left\lceil\frac{\Delta(G)+1}{2}\right\rceil\right\}$.

The following conjecture presented by Ivanco and Jendrol gave the exact value of the total edge of irregularity strength for arbitrary graph.

Conjecture 1.2. 5] For any graph $G$ which maximum vertex degree is $\Delta(G)$,

$$
\operatorname{tes}(G)=\max \left\{\left\lceil\frac{|E(G)|+2}{3}\right\rceil,\left\lceil\frac{\Delta(G)+1}{2}\right\rceil\right\} .
$$

The conjecture has been proved to be true for all trees, complete graphs except $K_{5}$, complete bipartite graphs, zig zag graphs, helms, closed helms, fans, tadpoles, armed crowns, webs, flowers, gears, sun flowers, and some other graphs. The tes of trees was given by Ivančo and Jendrol ([5]) while the tes 
for complete graphs and complete bipartite graphs were studied by Jendrol, et al. 6]. The tes of zig zag graphs was given by Ahmad in [1. In [7] and 8], Putra, et al. observed the tes of centralized uniform theta graphs and the tes of uniform theta graphs, respectively. In [9] and [10, Ratnasari, et al. gave the exact value of the tes of some ladder related graphs and arithmetic book graphs, respectively.

Based on 4, aside from total edge irregularity strength, there are many kinds of graph labeling. One among many kinds of labeling is a graceful labeling. We found an interesting work done by Solairaju, et al. [11] about the graceful mirror-staircase graphs. Some graphs that are presented in the paper are staircase graphs, double-staircase graphs, and mirror-staircase graphs. Therefore we are interested in observing the tes of the graphs. In this paper, we give the exact value of the total edge irregularity strength of the staircase, double-staircase and mirror-staircase graphs.

\section{Results}

In this paper, the first graph which the tes will be observed is the staircase graph. First, we denote the staircase graph of size $n \geq 1$ by $S C_{n}$ (see Figure 1). We have

$V\left(S C_{n}\right)=\left\{v_{i, j} \mid i=0,1, j=0,1,2, \ldots, n\right\} \cup\left\{v_{i, j} \mid i=2, \ldots, n, j=i-1, \ldots, n\right\}$

and $E\left(S C_{n}\right)$ which consists of edges as the following.

\begin{tabular}{lll}
\hline edges & $i$ & $j$ \\
\hline$v_{i, j} v_{i+1, j}$ & 0 & $0,1,2, \ldots, n$ \\
$v_{i, j} v_{i+1, j}$ & $1, \ldots, n-1$ & $i, \ldots, n$ \\
$v_{i, j} v_{i, j+1}$ & 0,1 & $0,1,2, \ldots, n-1$ \\
$v_{i, j} v_{i, j+1}$ & $2, \ldots, n$ & $i-1, \ldots, n-1$ \\
\hline
\end{tabular}

By a simple calculation we have that $\left|V\left(S C_{n}\right)\right|=\frac{1}{2}(n+1)(n+2)+n$ and $\left|E\left(S C_{n}\right)\right|=n(n+3)$.

Theorem 2.1. Let $S C_{n}$ be the staircase graph of order $n$. Then

$$
\operatorname{tes}\left(S C_{n}\right)=\left\lceil\frac{n(n+3)+2}{3}\right\rceil \text {. }
$$

Proof. It is clear that the maximum degree of the staircase graph is 2 for $n=1$ and 4 for otherwise. Therefore, by Theorem 1.1), we obtain

$$
\operatorname{tes}\left(S C_{n}\right) \geq\left\lceil\frac{n(n+3)+2}{3}\right\rceil \text {. }
$$

To complete the proof we show that $\operatorname{tes}\left(S C_{n}\right) \leq\left\lceil\frac{n(n+3)+2}{3}\right\rceil$, by showing that there exists a total edge irregularity $k$-labeling with $k=\left\lceil\frac{n(n+3)+2}{3}\right\rceil$. First, we 


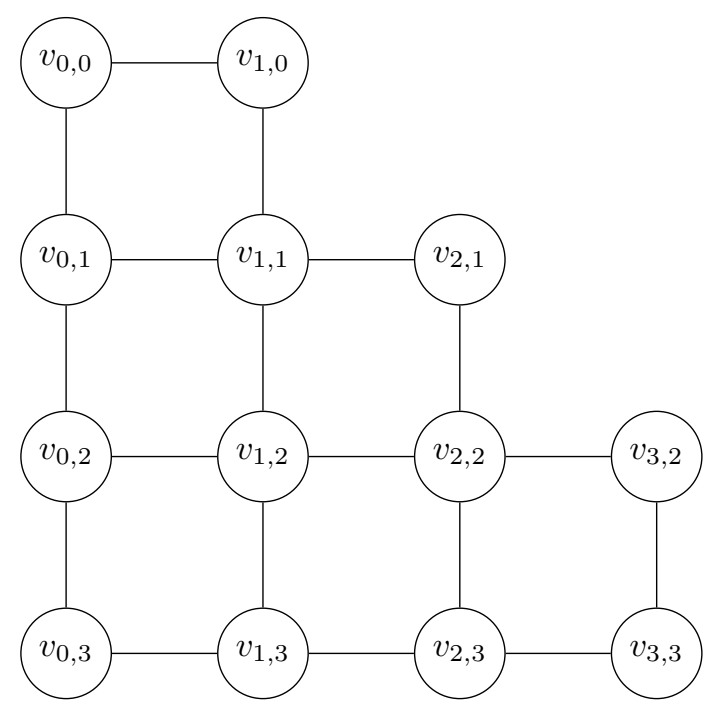

Figure 1. Staircase Graph $S C_{3}$

determine the biggest positive number $m$ such that

$$
\frac{m(m+1)}{2} \leq\left\lceil\frac{n(n+3)+2}{3}\right\rceil-1 .
$$

(Several $n$ and $m$ are listed on Table 1.)

\begin{tabular}{|c|c|c|c|c|c|c|c|c|c|}
\hline$n$ & $m$ & $n$ & $m$ & $n$ & $m$ & $n$ & $m$ & $n$ & $m$ \\
\hline 1 & 1 & 6 & 5 & 11 & 9 & 16 & 13 & 21 & 17 \\
\hline 2 & 2 & 7 & 6 & 12 & 10 & 17 & 14 & 22 & 18 \\
\hline 3 & 3 & 8 & 7 & 13 & 11 & 18 & 15 & 23 & 19 \\
\hline 4 & 3 & 9 & 8 & 14 & 12 & 19 & 16 & 24 & 20 \\
\hline 5 & 4 & 10 & 8 & 15 & 12 & 20 & 17 & 25 & 21 \\
\hline
\end{tabular}

TABLE 1.

We define a $k$-labeling

$$
f: V\left(S C_{n}\right) \cup E\left(S C_{n}\right) \rightarrow\left\{1,2, \ldots,\left\lceil\frac{n(n+3)+2}{3}\right\rceil\right\}
$$


to label the graph elements in the following way:

\begin{tabular}{ll}
\hline edges and vertices label & $i$ and $j$ \\
\hline$f\left(v_{i, 0}\right)=1$ & $i=0,1$ \\
\hline$f\left(v_{i, j}\right)=\frac{j^{2}+j+2}{2}$ & $i=0,1, \ldots, j+1$ \\
& $j=1,2, \ldots, m$ \\
\hline$f\left(v_{i, j} v_{i+1, j}\right)=i+j+1$ & $i=0,1, \ldots, j$ \\
& $j=0, \ldots, m-1$ \\
\hline$f\left(v_{i, j} v_{i+1, j}\right)=i+j+1$ & $i=0,1, \ldots, m-1$ \\
& $j=m$ \\
\hline$f\left(v_{i, j} v_{i, j+1}\right)=i+j+1$ & $i=0,1, \ldots, j+1$ \\
& $j=0, \ldots, m-1$ \\
\hline
\end{tabular}

We stop the process if $m=n$. If $m<n$, then we continue with

\begin{tabular}{ll}
\hline edges and vertices label & $i$ and $j$ \\
\hline$f\left(v_{i, j} v_{i+1, j}\right)=i+j+1$ & $i=m$ \\
\hline$f\left(v_{i, j}\right)=\left\lceil\frac{n(n+3)+2}{3}\right\rceil$ & $j=m$ \\
& $i=0, \ldots, j+1$ \\
& $j=m+1, \ldots, n$ \\
\hline$f\left(v_{i, j} v_{i, j+1}\right)=i+\frac{(m+3)(m+2)}{2}-\left\lceil\frac{n(n+3)+2}{3}\right\rceil$ & $i=0, \ldots, m+1$ \\
\hline$f\left(v_{i, j} v_{i+1, j}\right)=i+6+(m+k-1)(m+k+3)-$ & $i=0, \ldots, j$ \\
$2\left\lceil\frac{n(n+3)+2}{3}\right\rceil$ & $j=m+k$ \\
\hline$f\left(v_{i, j} v_{i, j+1}\right)=i+4+(m+k)(m+k+3)-$ & $i=0,1, \ldots, m+k+1$ \\
$2\left\lceil\frac{n(n+3)+2}{3}\right\rceil$ & $j=m+k$ \\
\hline$f\left(v_{i, j} v_{i+1, j}\right)=i+n^{2}+2 n+3-2\left\lceil\frac{n(n+3)+2}{3}\right\rceil$ & $j=1, \ldots, n-m-1$ \\
\hline & $i=n, 1, \ldots, n-1$
\end{tabular}


We then have the weights of the edges as follows:

\begin{tabular}{ll}
\hline weights & $i$ and $j$ \\
\hline$w t_{f}\left(v_{i, 0} v_{i+1,0}\right)=i+3$ & $i=0$ \\
\hline$w t_{f}\left(v_{i, 0} v_{i, 1}\right)=i+4$ & $i=0,1$ \\
\hline$w t_{f}\left(v_{i, j} v_{i+1, j}\right)=i+j^{2}+2 j+3$ & $i=0,1, \ldots, j$ \\
& $j=1,2, \ldots, m$ \\
\hline$w t_{f}\left(v_{i, j} v_{i, j+1}\right)=i+j^{2}+3 j+i+4$ & $i=0,1, \ldots, j+1$ \\
\hline$w t_{f}\left(v_{i, j} v_{i, j+1}\right)=i+\frac{j^{2}+j+2+(m+3)(m+2)}{2}$ & $j=1,2, \ldots, m-1$ \\
\hline$w t_{f}\left(v_{i, j} v_{i+1, j}\right)=i+6+(m+k-1)(m+k+3)$ & $i=0, \ldots, m+1$ \\
& $j=m$ \\
\hline$w t_{f}\left(v_{i, j} v_{i, j+1}\right)=i+4+(m+k)(m+k+3)$ & $j=m+k$ \\
& $k=1, \ldots, n-m-1$ \\
\hline$w t_{f}\left(v_{i, j} v_{i+1, j}\right)=i+n^{2}+2 n+3$ & $j=m+k, m+k+1$ \\
& $k=1, \ldots, n-m-1$ \\
\hline
\end{tabular}

Now we are going to consider the double staircase for the second observation. In this paper, we denote the double staircase (See Figure 2 of order $n$ by $D S C_{n}$. We have

$$
\begin{gathered}
V\left(D S C_{n}\right)=\left\{l_{i, j} \mid i=1,2, \ldots, n, j=i-1, \ldots, n\right\} \\
\cup\left\{r_{i, j} \mid i=1,2, \ldots, n, j=i-1, \ldots, n\right\}
\end{gathered}
$$

and $E\left(D S C_{n}\right)$ which consists of edges as given in the following table.

\begin{tabular}{lll}
\hline edges & $i$ & $j$ \\
\hline$l_{i, j} r_{i, j}$ & 1 & $0,1,2, \ldots, n$ \\
$l_{i+1, j} l_{i, j}$ & $1,2, \ldots, n-1$ & $1,2, \ldots, n$ \\
$r_{i, j} r_{i+1, j}$ & $1,2, \ldots, n-1$ & $1,2, \ldots, n$ \\
$l_{i, j} l_{i, j+1}$ & $1,2, \ldots, n$ & $i-1, \ldots, n$ \\
$r_{i, j} r_{i, j+1}$ & $1,2, \ldots, n$ & $i-1, \ldots, n$ \\
\hline
\end{tabular}

By a short calculation we have that $\left|V\left(D S C_{n}\right)\right|=n^{2}+3 n$ and $\left|E\left(D S C_{n}\right)\right|=$ $2 n^{2}+3 n-1$.

Theorem 2.2. Let $D S C_{n}$ be the double staircase graph of order $n$. Then

$$
\operatorname{tes}\left(D S C_{n}\right)=\left\lceil\frac{2 n^{2}+3 n+1}{3}\right\rceil .
$$

Proof. Similar to the staircase graph, we know that the maximum degree of the double staircase graph is 2 for $n=1$ and 4 for otherwise. Thus, by Theorem 


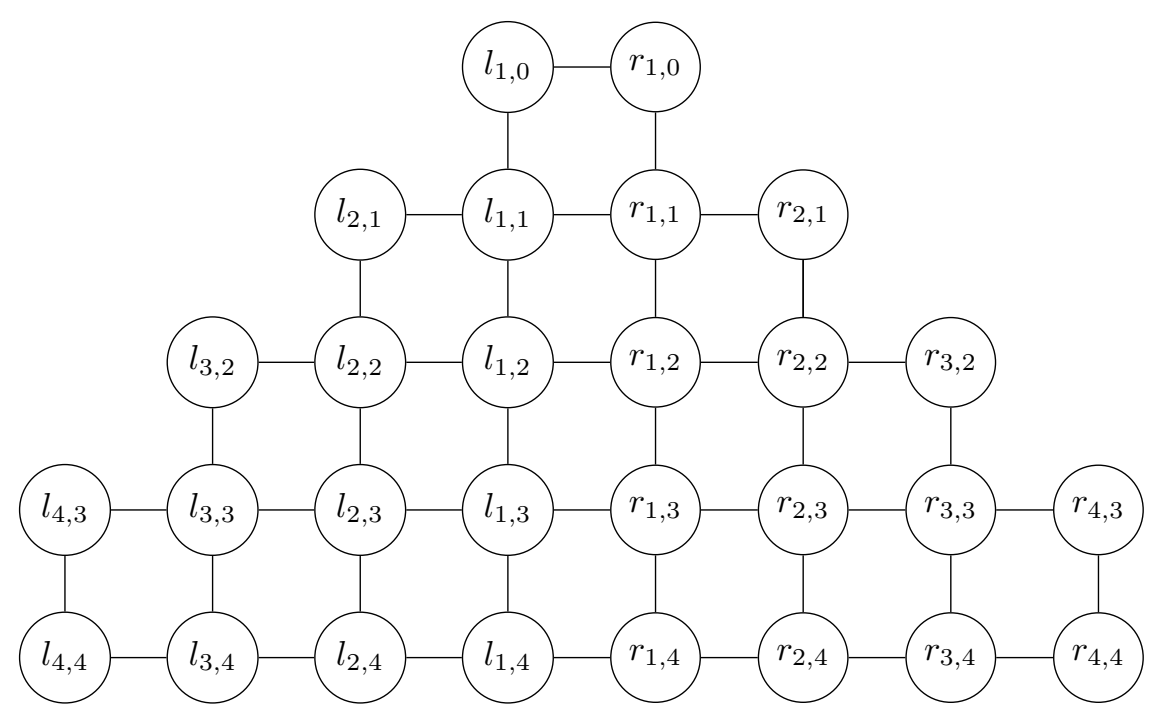

Figure 2. Double Staircase Graph $\mathrm{DSC}_{4}$

(1.1), we have

$$
\operatorname{tes}\left(D S C_{n}\right) \geq\left\lceil\frac{2 n^{2}+3 n+1}{3}\right\rceil
$$

Again, in order to converse the inequality, all we have to do is to show the existence of total edge irregularity $k$-labeling with $k=\left\lceil\frac{2 n^{2}+3 n+1}{3}\right\rceil$.

Let $k=\left\lceil\frac{2 n^{2}+3 n+1}{3}\right\rceil$, then we construct an

$$
f: V\left(D S C_{n}\right) \cup E\left(D S C_{n}\right) \rightarrow\{1,2, \ldots, k\}
$$


as a $k$-labeling in following manner:

\begin{tabular}{|c|c|}
\hline edges and vertices label & $i$ and $j$ \\
\hline$f\left(l_{i, j}\right)=\left\lceil\frac{2 j^{2}+3 j+1}{3}\right\rceil$ & $\begin{array}{l}i=1,2, \ldots, n \\
j=i-1, \ldots, n\end{array}$ \\
\hline$f\left(r_{i, j}\right)=\left\lceil\frac{2 j^{2}+3 j+1}{3}\right\rceil$ & $\begin{array}{l}i=1,2, \ldots, n \\
j=i-1, \ldots, n\end{array}$ \\
\hline$f\left(l_{i, j} r_{i, j}\right)=1$ & $i=1, j=0$ \\
\hline$f\left(l_{1, j} r_{1, j}\right)=\left(2\left\lceil\frac{j}{3}\right\rceil-1\right) j-\left\lceil\frac{j}{3}\right\rceil+2$ & $1 \leq j \leq n, j=1 \bmod 3$ \\
\hline$f\left(l_{1, j} r_{1, j}\right)=2\left\lceil\frac{j}{3}\right\rceil(j-1)+2$ & $1 \leq j \leq n, j=2 \bmod 3$ \\
\hline$f\left(l_{1, j} r_{1, j}\right)=2 j\left\lceil\frac{j}{3}\right\rceil-3\left\lceil\frac{j}{3}\right\rceil+j$ & $1 \leq j \leq n, j=0 \bmod 3$ \\
\hline $\begin{array}{l}f\left(l_{i+1, i} l_{i, i}\right)=i^{2}-2 i-\left\lceil\frac{i-4}{3}\right\rceil(i-1)+ \\
4\left\lceil\frac{i-1}{3}\right\rceil+4\end{array}$ & $1 \leq i \leq n-1, i=1 \bmod 3$ \\
\hline $\begin{array}{l}f\left(l_{i+1, i} l_{i, i}\right)=i^{2}-4 i-\left\lceil\frac{i-5}{3}\right\rceil(i-2)+ \\
8\left\lceil\frac{i-2}{3}\right\rceil+10\end{array}$ & $1 \leq i \leq n-1, i=2 \bmod 3$ \\
\hline$f\left(l_{i+1, i} l_{i, i}\right)=i\left(i-\left\lceil\frac{i-3}{3}\right\rceil\right)$ & $1 \leq i \leq n-1, i=0 \bmod 3$ \\
\hline $\begin{array}{l}f\left(l_{i+1, j} l_{i, j}\right)=\left\lceil\frac{2 j^{2}+3 j+1}{3}\right\rceil-3\left\lceil\frac{j-2}{3}\right\rceil \\
-i-1\end{array}$ & $\begin{array}{l}1 \leq i \leq n-1 \\
i+1 \leq j \leq n, j=2 \bmod 3\end{array}$ \\
\hline $\begin{array}{l}f\left(l_{i+1, j} l_{i, j}\right)=\left\lceil\frac{2 j^{2}+3 j+1}{3}\right\rceil-6\left\lceil\frac{j-1}{3}\right\rceil \\
+j-i-1\end{array}$ & $\begin{array}{l}1 \leq i \leq n-1 \\
i+1 \leq j \leq n, j=1 \bmod 3 \\
\text { or } j=0 \bmod 3\end{array}$ \\
\hline $\begin{array}{l}f\left(l_{i, j} l_{i, j+1}\right)=2(j+1)\left\lceil\frac{j-1}{3}\right\rceil+2\left\lceil\frac{j}{3}\right\rceil \\
-i+2\end{array}$ & $\begin{array}{l}i=1,2, \ldots, n \\
i-1 \leq j \leq n-1 \\
j=1 \bmod 3\end{array}$ \\
\hline$f\left(l_{i, j} l_{i, j+1}\right)=2 j\left\lceil\frac{j}{3}\right\rceil-i+2$ & $\begin{array}{l}i=1,2, \ldots, n \\
i-1 \leq j \leq n-1 \\
j=2 \bmod 3\end{array}$ \\
\hline$f\left(l_{i, j} l_{i, j+1}\right)=2(j+1)\left\lceil\frac{j}{3}\right\rceil-i+2$ & $\begin{array}{l}i=1,2, \ldots, n \\
i-1 \leq j \leq n-1 \\
j=0 \bmod 3\end{array}$ \\
\hline $\begin{array}{l}f\left(r_{i, i} r_{i+1, i}\right)=i^{2}-2 i-\left\lceil\frac{i-4}{3}\right\rceil(i-1)+ \\
4\left\lceil\frac{i-1}{3}\right\rceil+5\end{array}$ & $1 \leq i \leq n-1, i=1 \bmod 3$ \\
\hline $\begin{array}{l}f\left(r_{i, i} r_{i+1, i}\right)=i^{2}-4 i-\left\lceil\frac{i-5}{3}\right\rceil(i-2)+ \\
8\left\lceil\frac{i-2}{3}\right\rceil+11\end{array}$ & $1 \leq i \leq n-1, i=2 \bmod 3$ \\
\hline$f\left(r_{i, i} r_{i+1, i}\right)=i\left(i-\left\lceil\frac{i-3}{3}\right\rceil\right)+1$ & $1 \leq i \leq n-1, i=0 \bmod 3$ \\
\hline$f\left(r_{i, j} r_{i+1, j}\right)=\left\lceil\frac{2 j^{2}+3 j+1}{3}\right\rceil-j+i-1$ & $\begin{array}{l}1 \leq i \leq n-1 \\
i+1 \leq j \leq n, j=0 \bmod 3\end{array}$ \\
\hline$f\left(r_{i, j} r_{i+1, j}\right)=\left\lceil\frac{2 j^{2}+3 j+1}{3}\right\rceil-j+i+1$ & $\begin{array}{l}1 \leq i \leq n-1 \\
i+1 \leq j \leq n, j=1 \bmod 3 \\
\text { or } j=2 \bmod 3\end{array}$ \\
\hline$f\left(r_{i, j} r_{i, j+1}\right)=2(j+1)\left\lceil\frac{j-1}{3}\right\rceil+2\left\lceil\frac{j}{3}\right\rceil+i+1$ & $\begin{array}{l}i=1,2, \ldots, n \\
i-1 \leq j \leq n-1 \\
j=1 \bmod 3\end{array}$ \\
\hline$f\left(r_{i, j} r_{i, j+1}\right)=2 j\left\lceil\frac{j}{3}\right\rceil+i+1$ & $\begin{array}{l}i=1,2, \ldots, n \\
i-1 \leq j \leq n-1 \\
j=2 \bmod 3\end{array}$ \\
\hline $\begin{array}{l}f\left(r_{i, j} r_{i, j+1}\right)=2(j+1)\left\lceil\frac{j}{3}\right\rceil+i+1 \\
j=0 \bmod 3\end{array}$ & $\begin{array}{l}i=1,2, \ldots, n \\
i-1 \leq j \leq n-1\end{array}$ \\
\hline
\end{tabular}


Furthermore, to prove that $f$ is an edge irregular total $k$-labeling, we need to consider the weight of all edges in $D S C_{n}$. We obtain the weight of all edges, as follows:

\begin{tabular}{ll}
\hline weight & $i$ and $j$ \\
\hline$w t_{f}\left(l_{i, j} r_{i, j}\right)=3$ & $i=1, j=0$ \\
\hline$w t_{f}\left(l_{1, j} r_{1, j}\right)=2 j^{2}+2 j+2$ & $1 \leq j \leq n$ \\
\hline$w t_{f}\left(l_{i+1, i} l_{i, i}\right)=2 i^{2}+3 i+2$ & $1 \leq i \leq n-1$ \\
\hline$w t_{f}\left(l_{i+1, j} l_{i, j}\right)=2 j^{2}+2 j-i+2$ & $1 \leq i \leq n-1$ \\
& $i+1 \leq j \leq n$ \\
\hline$w t_{f}\left(l_{i, j} l_{i, j+1}\right)=2 j^{2}+4 j+5-i$ & $i=1,2, \ldots, n$ \\
& $i-1 \leq j \leq n-1$ \\
\hline$w t_{f}\left(r_{i, i} r_{i+1, i}\right)=2 i^{2}+3 i+3$ & $1 \leq i \leq n-1$ \\
\hline$w t_{f}\left(r_{i, j} r_{i+1, j}\right)=2 j^{2}+2 j+i+2$ & $1 \leq i \leq n-1$ \\
& $i+1 \leq j \leq n$ \\
\hline$w t_{f}\left(r_{i, j} r_{i, j+1}\right)=2 j^{2}+4 j+i+4$ & $i=1,2, \ldots, n$ \\
& $i-1 \leq j \leq n-1$ \\
\hline
\end{tabular}

We can list all of the weight of edges by the indices. Then, from the list, we obtain a sequence of integers from 3 up to $2 n^{2}+3 n+1$. It means that every different edge have different weight. It also implies that $f$ is an edge irregular total $k$-labeling with $k=\left\lceil\frac{2 n^{2}+3 n+1}{3}\right\rceil$.

Thus, we prove that $\operatorname{tes}\left(D S C_{n}\right)=\left\lceil\frac{2 n^{2}+3 n+1}{3}\right\rceil$.

For the last observation, we consider the mirror staircase graph of size $n \geq 1$ which we denote by $M S C_{n}$ (see Figure 3). We have

$$
\begin{aligned}
V\left(M S C_{n}\right)= & \left\{v_{i, j} \mid i=-1,0,1, j=0,1,2, \ldots, n\right\} \cup\left\{v_{i, j} \mid i=2, \ldots, n, j=i-1, \ldots, n\right\} \\
& \cup\left\{v_{i, j} \mid i=-2, \ldots,-n, j=-i-1, \ldots, n\right\}
\end{aligned}
$$

and $E\left(M S C_{n}\right)$ which consists of edges as listed below

\begin{tabular}{lll}
\hline edges & $i$ & $j$ \\
\hline$v_{i, j} v_{i+1, j}$ & $-1,0$ & $0,1,2, \ldots, n$ \\
$v_{i, j} v_{i+1, j}$ & $1, \ldots, n-1$ & $i, \ldots, n$ \\
$v_{i, j} v_{i+1, j}$ & $-2, \ldots,-n$ & $-i-1, \ldots, n$ \\
$v_{i, j} v_{i, j+1}$ & $-1,0,1$ & $0,1,2, \ldots, n-1$ \\
$v_{i, j} v_{i, j+1}$ & $2, \ldots, n$ & $i-1, \ldots, n-1$ \\
$v_{i, j} v_{i, j+1}$ & $-2, \ldots,-n$ & $-i-1, \ldots, n-1$. \\
\hline
\end{tabular}

By a routine calculation we have that $\left|V\left(M S C_{n}\right)\right|=n^{2}+4 n+1$ and $\left|E\left(M S C_{n}\right)\right|=n(2 n+5)$.

Theorem 2.3. Let $M S C_{n}$ be the mirror staircase graph of order $n$. Then

$$
\operatorname{tes}\left(M S C_{n}\right)=\left\lceil\frac{n(2 n+5)+2}{3}\right\rceil .
$$




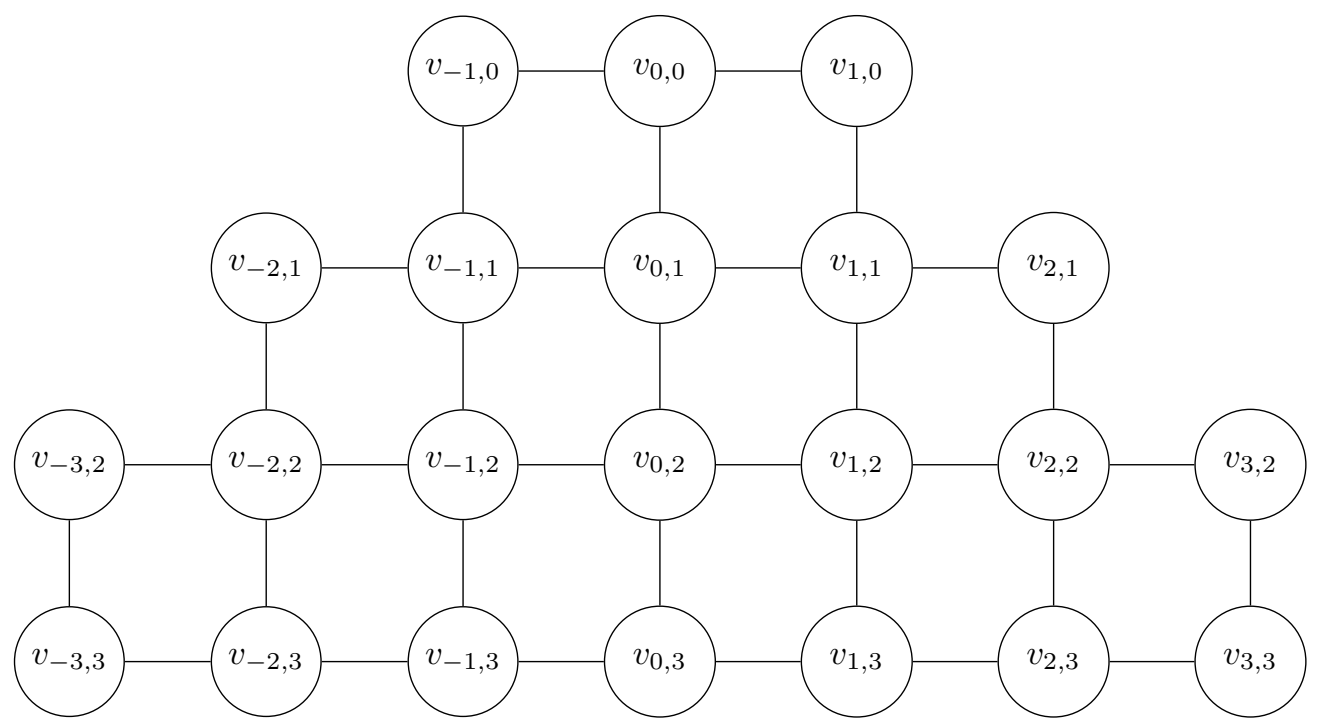

Figure 3. Mirror Staircase Graph $\mathrm{MSC}_{3}$

Proof. It is clear that the maximum degree of the staircase graph is 3 for $n=1$ and 4 for otherwise. Therefore, by Theorem (1.1), we obtain

$$
\operatorname{tes}\left(M S C_{n}\right) \geq\left\lceil\frac{n(2 n+5)+2}{3}\right\rceil \text {. }
$$

It remains to show that $\operatorname{tes}\left(M S C_{n}\right) \leq\left\lceil\frac{n(2 n+5)+2}{3}\right\rceil$ by proving that there exists a total edge irregularity $k$-labeling with $k=\left\lceil\frac{n(2 n+5)+2}{3}\right\rceil$. First, we determine the biggest positive number $m$ such that

$$
m^{2}+m+1 \leq\left\lceil\frac{n(2 n+5)+2}{3}\right\rceil-1 .
$$

(Several $n$ and $m$ are listed on Table 2)

\begin{tabular}{|c|c|c|c|c|c|c|c|c|c|c|c|c|}
\hline$n$ & $m$ & & $n$ & $m$ & & $n$ & $m$ & $n$ & $m$ & & $n$ & $m$ \\
\hline 1 & 1 & 6 & 5 & & 11 & 9 & 16 & 13 & 21 & 17 \\
2 & 2 & 7 & 6 & & 12 & 10 & 17 & 14 & 22 & 18 \\
3 & 2 & 8 & 6 & & 13 & 11 & 18 & 15 & 23 & 19 \\
4 & 3 & 9 & 7 & & 14 & 11 & 19 & 15 & 24 & 20 \\
5 & 4 & & 10 & 8 & & 15 & 12 & 20 & 16 & 25 & 20 \\
\hline
\end{tabular}

TABLE 2 . 
We define a $k$-labeling

$$
f: V\left(M S C_{n}\right) \cup E\left(M S C_{n}\right) \rightarrow\left\{1,2, \ldots,\left\lceil\frac{n(2 n+5)+2}{3}\right\rceil\right\}
$$

to label the graph elements in the following way:

\begin{tabular}{ll}
\hline edges and vertices label & $i$ and $j$ \\
\hline$f\left(v_{i, 0}\right)=1$ & $i=-1,0,1$ \\
\hline$f\left(v_{i, j}\right)=j^{2}+j+1$ & $i=-(j+1), \ldots, j+1$ \\
& $j=1,2, \ldots, m$ \\
\hline$f\left(v_{i, j}\right)=\left\lceil\frac{n(2 n+5)+2}{3}\right\rceil$ & $i=-(j+1), \ldots, j+1$ \\
& $j=m+1, \ldots, n-1$ \\
\hline$f\left(v_{i, n}\right)=\left\lceil\frac{n(2 n+5)+2}{3}\right\rceil$ & $i=-n, \ldots, n$ \\
\hline$f\left(v_{i, j} v_{i+1, j}\right)=i+2 j+2$ & $i=-(j+1), \ldots, j$ \\
& $j=0, \ldots, m$ \\
\hline$f\left(v_{i, j} v_{i, j+1}\right)=i+2 j+2$ & $i=-(j+1), \ldots, j+1$ \\
& $j=0, \ldots, m-1$.
\end{tabular}

We stop the process if $m=n$. If $m<n$, then we continue with

\begin{tabular}{ll}
\hline edges and vertices label & $i$ and $j$ \\
\hline$f\left(v_{i, j} v_{i, j+1}\right)=i+m^{2}+5(m+1)-\left\lceil\frac{n(2 n+5)+2}{3}\right\rceil$ & $i=-(j+1), \ldots, j+1$ \\
& $j=m$ \\
\hline$f\left(v_{i, j} v_{i+1, j}\right)=i+2\left((m+k+1)^{2}-\left\lceil\frac{n(2 n+5)+2}{3}\right\rceil\right)$ & $i=-(j+1), \ldots, j$ \\
+2 & $j=m+k$ \\
& $k=1, \ldots, n-m-1$ \\
& $k \geq 1$ \\
\hline$f\left(v_{i, j} v_{i, j+1}\right)=i+2\left((m+k+1)^{2}-\left\lceil\frac{n(2 n+5)+2}{3}\right\rceil\right)$ & $i=-(j+1), \ldots, j+1$ \\
$+2(m+k)+4$ & $j=m+k$ \\
& $k=1, \ldots, n-m-1$ \\
& $k \geq 1$ \\
\hline$f\left(v_{i, j} v_{i+1, j}\right)=i+2\left((n+1)^{2}-\left\lceil\frac{n(2 n+5)+2}{3}\right\rceil\right)+1$ & $i=-n, \ldots, n-1$ \\
& $j=n$
\end{tabular}


For arbitrary edge $x y$, the weight of $x y$ is $w t_{f}(x, y)=f(x)+f(x, y)+f(y)$. Therefore, we obtain the weight of all edges as follows:

\begin{tabular}{ll}
\hline weight & $i$ and $j$ \\
\hline$w t_{f}\left(v_{i, j} v_{i+1, j}\right)=i+4$ & $i=-1,0$ \\
& $j=0$ \\
\hline$w t_{f}\left(v_{i, j} v_{i+1, j}\right)=i+2 j^{2}+4 j+4$ & $i=-(j+1), \ldots, j$ \\
\hline$w t_{f}\left(v_{i, j} v_{i+1, j}\right)=i+2(m+k+1)^{2}+2$ & $j=1,2, \ldots m$ \\
\hline$w t_{f}\left(v_{i, j} v_{i+1, j}\right)=i+2(n+1)^{2}+1$ & $i=-(j+1), \ldots, j$ \\
\hline$w t_{f}\left(v_{i, j} v_{i, j+1}\right)=i+6$ & $j=m+k$ \\
\hline$w t_{f}\left(v_{i, j} v_{i, j+1}\right)=i+2 j^{2}+6 j+6$ & $k=1, \ldots, n-m-1$ \\
\hline$w t_{f}\left(v_{i, j} v_{i, j+1}\right)=i+2(m+k+1)(m+k+2)+2$ & $i=-n, \ldots, n-1$ \\
& $j=n$ \\
\hline & $i=-1,0,1$ \\
& $j=0$ \\
& $i=-(j+1), \ldots, j+1$ \\
& $j=1,2, \ldots, m$ \\
\hline
\end{tabular}

We can see that the weight of edge $x y$, for each $x y \in E\left(M S C_{n}\right)$ is all different.

\section{ACKNOWLEDGMENTS}

The author would like to thank the anonymous referees for their constructive comments.

\section{REFERENCES}

1. A. Ahmad, On the total edge irregularity strength of zigzag graphs, Australasian Journal of Combinatorics, 54, (2012), 141-149.

2. M. Bača, S. Jendrol, M. Miller, J. Ryan, On irregular total labeling. Discrete Math, 307, (2007), 1378-1388.

3. G. Chartrand, M.S. Jacobson, J. Lehel, O.R. Oellermann, S. Ruiz, F. Saba, Irregular networks, Congr. Numer. 64, (1988), 355-374.

4. J.A. Gallian, A dynamic survey of graph labeling. The Electronic Journal of Combinatorics, 18, (2015), 247-252.

5. J. Ivančo, S. Jendrol, The total edge irregularity strength of trees, Discuss. Math. Graph Theory, 26, (2006), 449-456.

6. S. Jendrol, J. Miskuf, R. Sotak, Total edge irregularity strength of complete graphs and complete bipartite graphs, Discrete Mathematics, 310(3), (2010), 400-407.

7. R.W. Putra, Y. Susanti, On total edge irregularity strength of centralized uniform theta graphs, AKCE International Journal of Graphs and Combinatorics, 15(1), (2018), 7-13.

8. R.W. Putra, Y. Susanti, The total edge irregularity strength of uniform theta graphs, Journal of Physics: Conference Series 1097(1), 012069 (2018). 
9. L. Ratnasari, Y. Susanti, Total edge irregularity strength of ladder related graphs, AsianEuropean Journal of Mathematics, doi:10.1142/S1793557120500722.

10. L. Ratnasari, S. Wahyuni, Y. Susanti, D. Junia Eksi Palupi and B. Surodjo, Total edge irregularity strength of arithmetic book graphs, Journal of Physics: Conference Series 1306(1), 012032 (2019).

11. A. Solairaju, A.M. Arockiasamy, Graceful mirror-staircase graphs, Int. J. Contemp. Math. Sciences, 5(49), (2010), 2433 - 2441.

12. W.D. Wallis, Magic graphs. Boston: Birkhãuser (2011). 